\title{
Interactions between Endostatin and Vascular Endothelial Growth Factor (VEGF) and Inhibition of Choroidal Neovascularization
}

\author{
Xun Wang ${ }^{1}$, Xun Xu ${ }^{1, *}$, Luosheng Tang ${ }^{2}$ Shiping Song ${ }^{3}$ and Chunhai Fan ${ }^{3}$ \\ 1 Shanghai Jiaotong University Affiliated First People's Hospital, Shanghai 200080, P.R. China \\ 2 Second Xiangya Hospital of Central South University, Changsha 410011, P.R. China \\ 3 Shanghai Institute of Applied Physics, Chinese Academy of Sciences, Shanghai 201800, P.R. China \\ * Author to whom correspondence should be addressed. E-mail: wangx200508@sina.com
}

Received: 03 January 2007 / Accepted: 16 January 2007 / Published: 31 January 2007

\begin{abstract}
This study was designed to evaluate the inhibitory effect of endostatin on choroidal neovascularization $(\mathrm{CNV})$ in laser-induced rat model. Choroidal neovascularization was induced in Brown Norway $(\mathrm{BN})$ rats by diode-laser photocoagulation. Rats were randomly divided into five groups (10 animals in each group): endostatin $20 \mathrm{mg} / \mathrm{kg}$ group, endostatin $10 \mathrm{mg} / \mathrm{kg}$ group, laser injury group, normal saline group and blank control group. The animals were treated with endostatin, normal saline, laser injury or without treatment on day 0 to day 13 after laser photocoagulation. On day 7 and 14 after laser photocoagulation, the CNV formation was assessed by fluorescein angiography and histopathological analysis. VEGF expression in retina was determined by immunohistochemical assay. In two endostatin groups, the incidence of CNV formation and the intensity of fluorescein leakage were reduced compared with the two control groups. No significant difference was found between laser injury group and normal saline group. The expression of VEGF reached peak at day 7 and then decreased from day 14 after photocoagulation. The expression of VEGF was significantly reduced in the two endostain groups than laser injury group in a dose-dependent way. Endostatin can inhibit the formation of experimental CNV in the rat. Down-regulation of VEGF expression could be one of the mechanisms underlying the inhibition of CNV by endostatin.
\end{abstract}

Keywords: horoidal neovascularization; Animal model; Laser; Endostatin; VEGF 


\section{Introduction}

Choroidal neovascularization $(\mathrm{CNV})$ is the common pathogenesis underlying age-related macular degeneration (AMD), central exudative chorioretinopathy, high myopia and other eye diseases. These diseases, especially AMD which is the major cause of blindness in the old people above 50 years of age, can result in severe impairment of vision. [1] In the population of more than 65 years old, $25 \%$ of the patients have the clinical features of AMD. And choroidal neovascularization is seen in $1.7 \%$ of these patients. The visual acuity of such patients may reduce to lower than 0.1 within 2 years. About $80 \%$ of these patients will eventually become blind. [2] At present, researchers are actively searching for pathway of effective CNV drug therapy. Endostatin attracts much attention in anti-tumor researches due to its potent inhibition of angiogenesis. It is a highly promising therapeutic protein for inhibition of angiogenesis. Considering the process of CNV formation is similar to tumor angiogenesis, we have studied the inhibition on experimental CNV and presented the results in the following.

\section{Results}

\subsection{FFA}

Of the 1139 laser spots observed by the 2 doctors, only 42 had different grading of fluorescein leakage. And the difference for all the spots was within 1 level. The higher grade was adopted for these 42 spots. At day 7 and day 14 following photocoagulation, the leakage rate by group was: laser injury group $40 \%$ and $67.82 \%$; normal saline group $38 \%$ and $70.19 \%$; endostatin group I $32.35 \%$ and $44.44 \%$; endostatin group II $9.61 \%$ and $23.64 \%$ respectively. The intensity of fluorescein leakage was presented in tables 1 and 2. There was no significant difference between laser injury group and normal saline group. In contrast, in the 2 endostatin groups, the number of leak spots in the ocular fundus of $\mathrm{BN}$ rats was less and leak intensity was weaker. Furthermore, the difference became more significant as duration of endostatin treatment lasted longer, and in a dose-dependent way (Figures 1 and 2). Fluorescein angiography of ocular fundus in normal BN rats did not show fluorescein leakage (Figure $3)$.

Table 1. Intensity of fluorescein leakage at Day 7 in BN rats assessed by FFA

\begin{tabular}{llllll}
\hline \multirow{2}{*}{ Group } & \multicolumn{3}{c}{ Intensity of fluorescein leakage } & \multirow{2}{*}{ Total } \\
\cline { 2 - 5 } & Grade 0 & Grade 1 & Grade 2 & Grade 3 & \\
\hline Laser injury & 99 & 24 & 27 & 15 & 165 \\
Normal saline & 93 & 24 & 21 & 12 & 150 \\
Endostatin group I & 138 & 24 & 24 & 18 & 204 \\
Endostatin group II & 141 & 9 & 6 & 0 & $156^{*}$ \\
\hline
\end{tabular}

NB: a) The difference of the overall grades of leakage intensity among the 4 groups was statistically significant $(\mathrm{Hc}=45.01$, $\mathrm{P}=0.000<0.01$ )

b) * : The difference of grades of leakage intensity between laser injury group, normal saline group and endostatin group I was not statistically significant. But the difference was statistically significant when these 3 groups compared with endostatin group II separately $(\mathrm{P}<0.01)$. 
Table 2. Intensity of fluorescein leakage at Day 14 in BN rats assessed by FFA

\begin{tabular}{llllll}
\hline \multirow{2}{*}{ Group } & \multicolumn{3}{l}{ Intensity of fluorescein leakage } & \multirow{2}{*}{ Total } \\
\cline { 2 - 5 } & Grade 0 & Grade 1 & Grade 2 & Grade 3 & \\
\hline Laser injury & 37 & 15 & 23 & 40 & 115 \\
Normal saline & 31 & 17 & 21 & 35 & 104 \\
Endostatin group I & 75 & 13 & 21 & 26 & $135^{*}$ \\
Endostatin group II & 84 & 11 & 12 & 3 & $110^{*}$ \\
\hline
\end{tabular}

NB: a) The difference of the overall grades of leakage intensity among the 4 groups was statistically significant ( $\mathrm{Hc}=70.217, \mathrm{P}=0.000<0.01)$

b) $* \square$ The difference of grades of leakage intensity between laser injury group and normal saline group was not statistically significant. But the difference between other groups was all statistically significant $(\mathrm{P}<0.01)$.
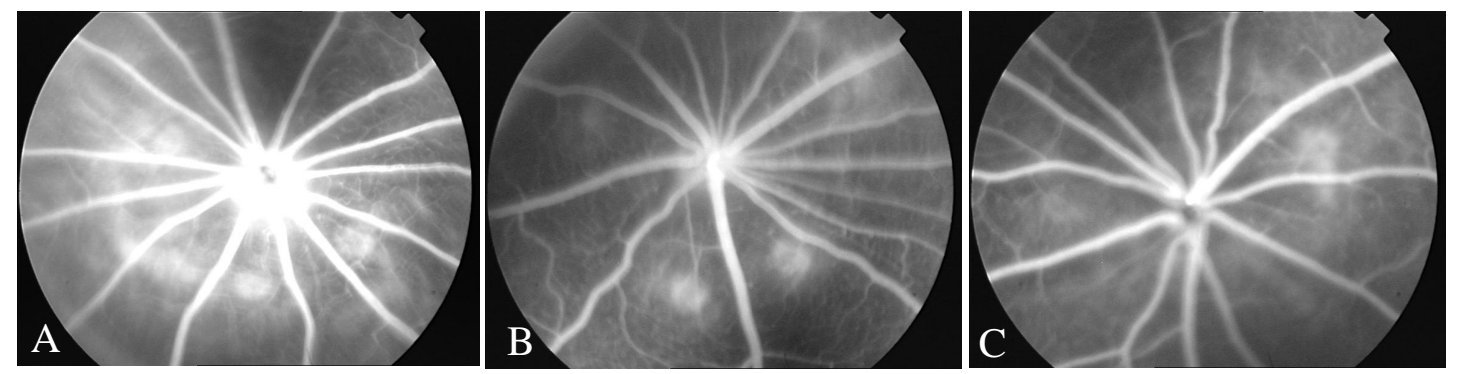

Figure 1. At day 7 after photocoagulation, FFA showed that A. laser injury group: mild fluorescein leakage was seen in laser spots of BN rats; B. endostatin group I: 2 laser spots with mild leakage can be seen; C. endostatin group II: no apparent fluorescein leakage was observed.
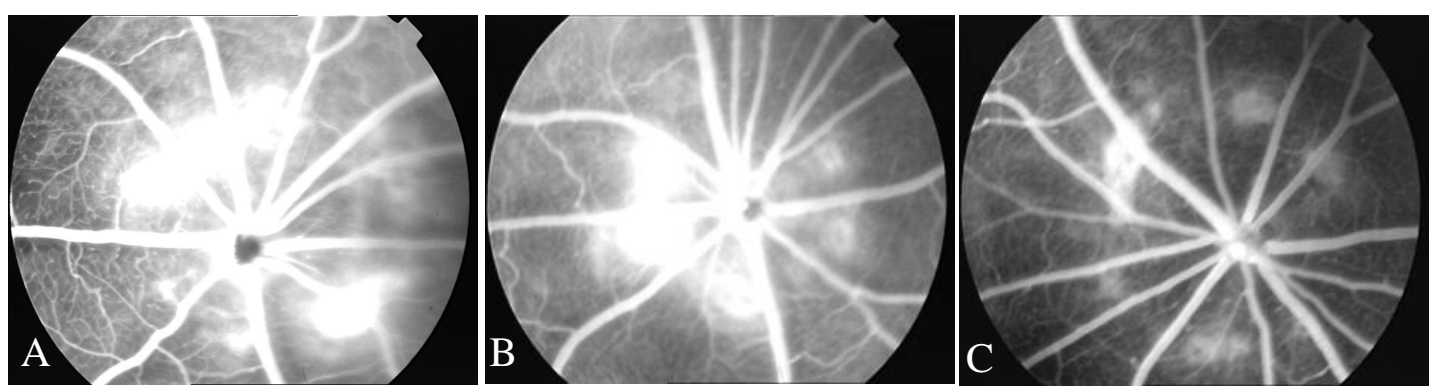

Figure 2. At day 14 after photocoagulation, FFA showed that A. laser injury group: obvious fluorescein leakage was observed in laser spots, high fluorescence was evident at early stage and fluorescent spots diffused and persisted at late stage of angiography; B. endostatin group I: 2 spots with severe leakage and 1 with moderate leakage; C. endostatin group II: only one spot of moderate leakage was seen.

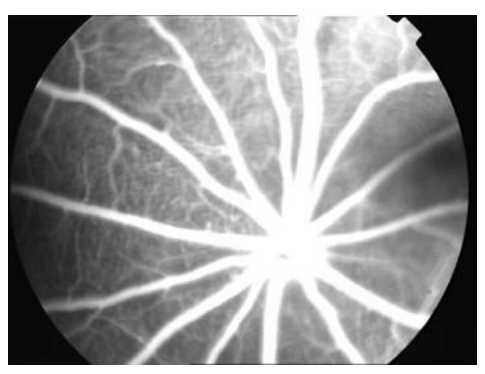

Figure 3. No fluorescein leakage was observed under FFA in normal BN rats 


\subsection{Histopathological examination}

The tissue structure of every layer of retina and choroid was well-defined and intact in normal $\mathrm{BN}$ rats (Figure 4A). At day 7 after photocoagulation, the retina at photocoagulation spot became thicker with deranged cell organization. The laser spots became thicker and organized with hyperplasia of a number of fibroblasts and a little CNV formation (Figure 4B). At day 14 after photocoagulation, the structure of retina was destructed. CNV broke through Bruch membrane and extended sprout-like toward underside of retina with huge hyperplasia of fibroblasts, as well as hyperplasia and migration of chromocytes (Figure 4C).

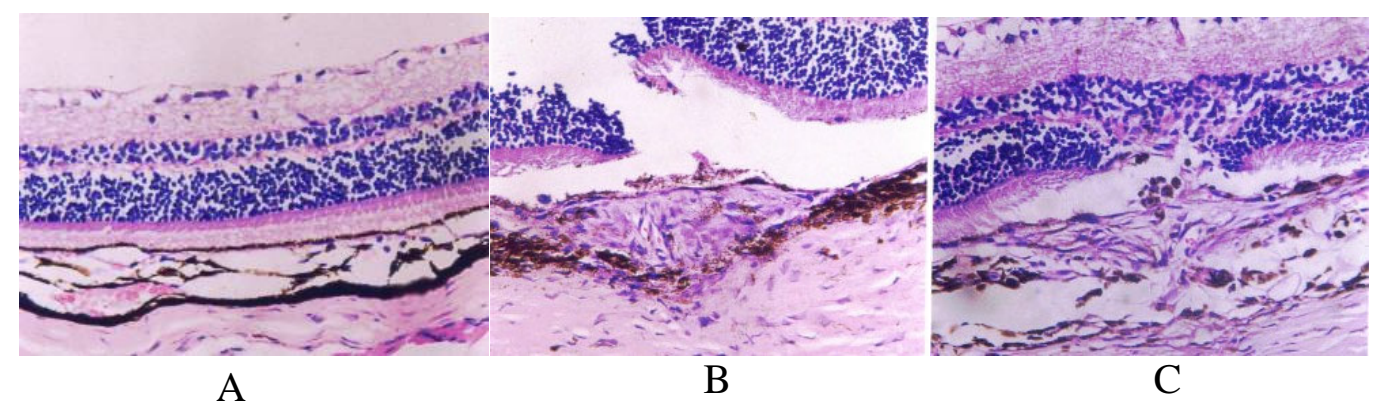

Figure 4. A. The tissue structure of every layer of retina and choroid is well-defined and intact in normal $\mathrm{BN}$ rats. B. At day 7 after photocoagulation, laser spots became thicker and organized with hyperplasia of a number of fibroblasts and a few CNV formation. C. At day 14 after photocoagulation, a lot of CNV formation broke through Bruch membrane and extended sprout-like toward underside of retina with hyperplasia and migration of chromocytes, as well as huge hyperplasia of fibroblasts (indicated by arrowhead).

\subsection{Expression of VEGF and bFGF $m R N A$}

No expression of VEGF was detected in the retina of normal BN rats in blank control group. Weak positive expression of VEGF was detected in partial pigment epithelium of retina. At day 7 after photocoagulation, the intensity of VEGF expression in retina of $\mathrm{BN}$ rats was higher in endostatin group I than that of endostatin group II with significant difference. The optical density score was significantly different between the two endostatin groups at day 7 and day 14 after photocoagulation (Table 3, Figure 5).

Table 3. VEGF expression in the retina of BN rats at different time points by group

\begin{tabular}{llllll}
\hline & & \multicolumn{3}{c}{ Optical density score } & \multirow{2}{*}{$\mathrm{P}_{1}^{*}$} \\
\cline { 2 - 5 } Time & Blank control & Laser injury & Endostatin group I & Endostatin group II & \\
\hline Day 7 & $22.9821 \pm 5.59801$ & $157.1627 \pm 8.96718$ & $114.1433 \pm 10.64936$ & $76.6473 \pm 11.2825$ & 0.00 \\
Day 14 & $22.9821 \pm 5.59801$ & $147.5433 \pm 10.24009$ & $100.7453 \pm 9.45678$ & $54.8053 \pm 7.28959$ & 0.00 \\
$\mathrm{P}_{2}$ & - & 0.01 & 0.00 & 0.00 & - \\
\hline NB: $\mathrm{P}_{1}$ : The overall difference of optical density score between groups was significant at the same time point. & \\
*: The difference of optical density score between any two groups was significant at the same time point. \\
P $\mathrm{P}_{2}$ : The optical density score was significantly different between different time points in all groups except blank control \\
group.
\end{tabular}




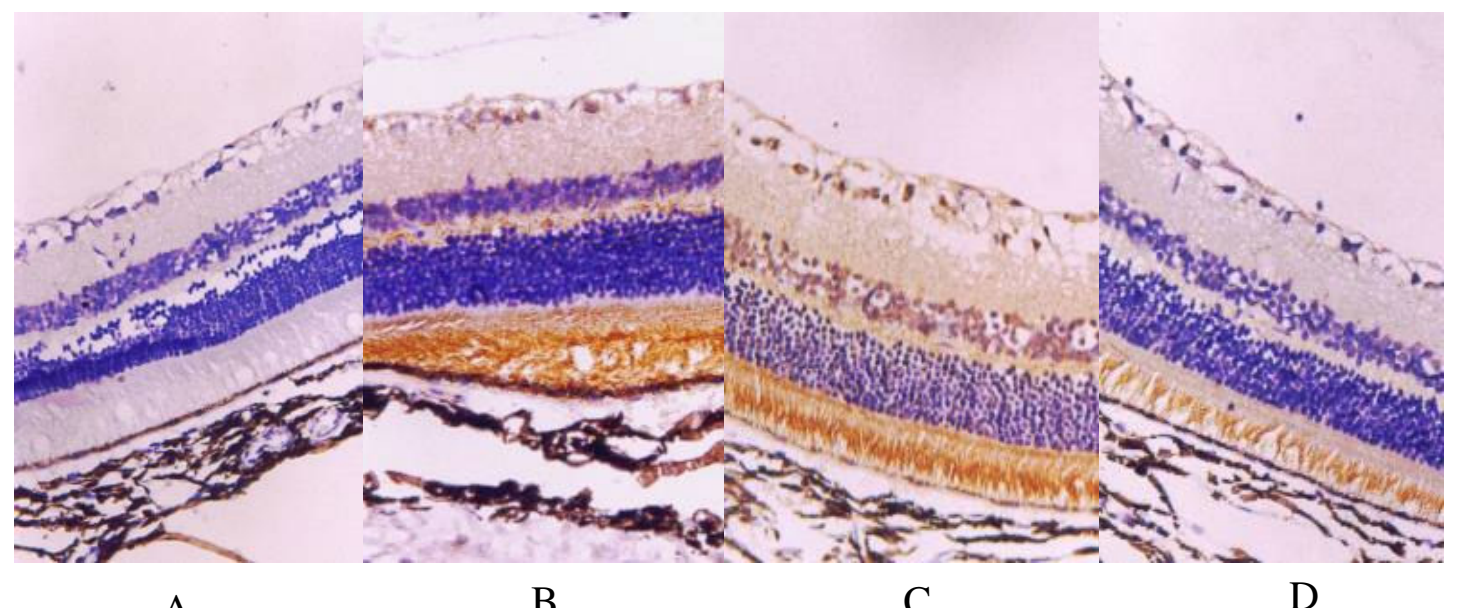

A

B

$\mathrm{C}$

$\mathrm{D}$

Figure 5. A: negative expression of VEGF in the retina of BN rats; B, C, D: immunohistochemical staining results of retina in $\mathrm{BN}$ rats 7 days after photocoagulation in laser injury group, endostatin group I and endostatin group II respectively.

\section{Discussion}

Choroidal neovascularization is characterized by choroidal neovascularization protruding through Bruch membrane, fibrous tissue and blood vessel proliferation formed in the space under retina which was caused by various factors. The resulted cascade of pathogenesis including exudation, hemorrhage, and organization are the principal cause of vision loss in various fundus diseases such as age-related macular degeneration. [3] The available clinical treatment modalities targeting CNV include laser treatment, photodynamic therapy, surgical intervention and radiation therapy, etc. All of these experimental treatment modalities have limitations with an unacceptably inadequate efficacy and high recurrence rate. Consequently, investigators have attempted to develop new modalities for treatment of CNV. It is well-known that neovascularization is due to the dysequilibrium between stimulators and inhibitors regulating angiogenesis and the tendency to angiogenesis. Therefore, one of the major strategies in drug therapy development is to reinforce the inhibitors of neovascularization.

Endostatin is a kind of angiogenesis inhibitor isolated and purified by O'Reilly et al from mouse endothelioma in Harvard University Medical School in 1997. [4] The molecular weight of endostatin is $20 \mathrm{kD}$. It is the hydrolytic fragment from the end of collagen X VIIIC. The tumor inhibition rate of endostatin in the treatment of Lewis lung cancer was $97 \%$ at dose of $10 \mathrm{mg} / \mathrm{kg} / \mathrm{day}$, and up to $99 \%$ at dose of $20 \mathrm{mg} / \mathrm{kg} /$ day. It has no toxicity or drug resistance. It is one of the most potent angiogenesis inhibitors discovered so far. In vitro studies, endostatin can specifically inhibit the proliferation and migration and induce apoptosis of vascular endothelial cells. [5, 6] Endostatin can also inhibit angiogenesis in various animal models. Studies [4] confirmed that endostatin had potent inhibitory effect on the neovascularization in chick chorioallantoic membrane, but without effect on the chick embryo per se. Mori et al [7] found that the formation and development of CNV was inversely correlated with serum endostatin level. He confirmed for the first time that systemically use of endostatin can inhibit intraocular neovascularization, and believed that daily injection of adequate endostatin is more effective than transgenic endostatin therapy by means of various vectors. 
The incidence of $\mathrm{CNV}$ is about $60 \%-100 \%$ in the retina of $\mathrm{BN}$ rats after treatment with laser photocoagulation. [8-12] Generally, CNV develops 7 days after photocoagulation, reaches peak 10 to 14 days after photocoagulation, and almost remains stable afterwards. CNV shrinks within 6 months. This study established CNV model in the BN rats after treatment with laser photocoagulation for 14 days. The success rate of modeling was $67.82 \%$. Endostatin was injected intra-abdominally daily at the same dose as that used by O'Reilly et al until day 13 after photocoagulation. Meanwhile, all BN rats in each group were studied by FFA and light microscopic examination to observe the inhibition of CNV by endostatin. Results showed that there was no significant difference between laser injury group and normal saline group. However, $\mathrm{CNV}$ formation in the ocular fundus of $\mathrm{BN}$ rats was reduced in the two endostatin treatment groups. The intensity of fluorescein leakage was relatively weaker in a dosedependent way. At day 7 after photocoagulation, lower dose endostatin showed tendency of CNV inhibition, but the difference was not significant. At day 14 following photocoagulation, the formation of $\mathrm{CNV}$ and leakage intensity were significantly inhibited. But higher dose endostatin had showed significant inhibition on CNV since day 7 after photocoagulation. Our study indicates that adequate endostatin can effectively inhibit the formation and development of CNV. This is in accordance with the findings reported by other authors [7,13].

The action mechanism of endostatin is not clear yet. Relevant studies indicate that endostatin can inhibit the proliferation and migration of endothelial cells and the neovascularization induced by VEGF. [14-18] VEGF plays a key role in the formation of CNV. [5] This study has confirmed with immunohistochemical assay that VEGF expression significantly increased in BN rat models induced by laser photocoagulation. The level of expression reached peak at day 7. The optical density score was 7 times that of normal retina tissue. At day 14, the level of VEGF expression began to decrease. In addition to the increase of expression amount, expression scope of VEGF in retina was also significantly wider than that in normal $\mathrm{BN}$ rats. The expression was mainly in the pigment epithelium layer, cone and rod cells layer, outer limiting membrane, external plexiform layer, internal granular layer and ganglion cell layer of retina. The results of other authors are essentially in consistent with our findings. [19-21] Jia et al [17] confirmed that endostatin acts by blocking the pathway of VEGF/receptor. In this study we found that VEGF expression in the retina of endostatin-treated BN rats significantly decreased compared with laser injury group. The decrease was dose-dependent. This suggests that endostatin may act not only by blocking the binding of VEGF to its specific receptor, but also by down-regulation of VEGF expression.

$\mathrm{CNV}$ is the major cause of blindness in the elderly. CNV formation is associated with various factors. VEGF plays an important role in the pathogenesis. Endostatin is one of the most promising therapeutic proteins in current antineovascularization therapy. It is very important to explore its inhibitory effect on CNV and its relation to VEGF. The results of this study indicate that endostatin can effectively inhibit laser-induced experimental CNV formation in BN rat models. Down-regulation of VEGF expression may be one of the mechanisms underlying the inhibition of CNV by endostatin. The findings may provide a new approach for further studies, and provide additional rationale for prevention and treatment of $\mathrm{CNV}$ in clinical practice. 


\section{Experimental}

\subsection{Reagents and equipments}

Recombinant human endostatin (Rongchang Bioengineering Co., Ltd, Yantai, Shandong Province, China); Rabbit anti-VEGF multiclonal antibody (Boshide Bioengineering Co., Ltd, Wuhan Hubei Province, China); SABC immunohistochemical staining kit (Boshide Bioengineering Co., Ltd, Wuhan Hubei Province, China); Diaminobenzidine (DAB) chromogen (Maixin Bioengineering Co., Ltd, Fujian Province, China)

Semiconductor laser (QUANTEL MEDICAL Co.); Image analysis system TM-2000 (Beihang Company)

\subsection{Procedures}

\subsubsection{Preparation of animal models}

Fifty male BN rats (100 eyes) were provided by Institute of Experimental Animals, Chinese Academy of Medical Sciences. Body weight of the animals was about $220 \mathrm{~g}$. Forty of the animals (80 eyes) were assigned to experimental groups, 10 animals (20 eyes) were in control group. The BN rats in experimental groups were anesthetized ( $3 \%$ pentobarbital by intra-abdominal injection at $40 \mathrm{mg} / \mathrm{kg}$ ) and $0.5 \%$ tropicamide was used for mydriasis. Semiconductor laser (wave length $532 \mathrm{~nm}$ ) was used to conduct retina photocoagulation surrounding optic papilla but spare the blood vessels. About 10 spots were made in each eye by laser at output power of $200 \mathrm{mw}$. The diameter of the light spot was $50 \mu \mathrm{m}$. Exposure time was $0.05 \mathrm{~s}$. The photocoagulation process was made to the extent of air bubble occurrence.

\subsubsection{Animal assignment}

The $40 \mathrm{BN}$ rats (80 eyes) without serious hemorrhage of ocular fundus or vitreous body by fundus examination after photocoagulation were randomized into 4 groups: endostatin group II, endostatin group I, normal saline group and laser injury group (10 animals / group). From day 0 to day 13 after photocoagulation, the animals in the first 3 groups were treated by intra-abdominal injection of endostatin $20 \mathrm{mg} / \mathrm{kg}, 10 \mathrm{mg} / \mathrm{kg}$ or normal saline $1.2 \mathrm{ml}$ respectively. But the animals in laser injury group did not receive any treatment after photocoagulation. The BN rats $(n=10)$ without laser photocoagulation or intra-abdominal injection were considered as blank control group.

\subsubsection{Fundus fluorescein angiography (FFA)}

BN rats were examined by FFA at Day 7 and Day 14 after photocoagulation. Specifically, BN rats were anesthetized and pupils were dilated. Continuous photography was conducted for 30 minutes immediately after $10 \%$ fluorescein sodium $(0.5 \mathrm{ml} / \mathrm{kg})$ was injected intra-abdominally to record the leakage of fluorescein. The leakage rate of fluorescein was calculated according to the following formula: leakage rate of fluorescein $=$ leak spots $(n) /$ observed laser spots $(n) \times 100 \%$. During late stage of intravenous injection, the intensity of fluorescein leakage was classified into 4 grades with 
reference to Takebana's method [22]: no leakage (Grade 0), mild leakage (Grade 1), moderate leakage (Grade 2) and severe leakage (Grade 3). The grading of fluorescein leakage was assessed by 2 experienced doctors specialized in ocular fundus diseases by double-blind manner.

\subsubsection{Light microscopic examination}

At day 7 and day 14 after photocoagulation, BN rats were sacrificed by intra-abdominal injection of overdose of $3 \%$ pentobarbital. Eyes of the killed animals were removed and embedded with paraffin. The continuous sections were prepared from the embedded samples sagittally as parallel to the optic nerve with section thickness of $6 \mu \mathrm{m}$. The paraffin sections were processed by HE stain and prepared for VEGF immunohistochemical assay (according to the instructions for the test kit).

\subsubsection{Image analysis}

The sections were examined with TM-2000 image analysis system under visual field of $400 \times$ magnification. Five visual fields were selected randomly from each section for analysis of the staining results. The mean of optical density score was calculated.

\subsubsection{Statistical analysis}

The intensity of fluorescein leakage was compared by rank sum test. The VEGF expression between groups at the same time point was compared by variance analysis and q test. The VEGF expression at different time points within the same group was analyzed by 2 -sample t-test to compare the means in a block design. The significance level was defined as $\mathrm{P}<0.05$.

\section{Acknowledgments}

This work was supported by National Natural Science Foundation of China.

\section{References}

1. Hooper, C. Y.; Guymer, R. H. New treatments in age-related macular degeneration. Clin. Experiment Ophthalmol. 2003, 31(5), 376-91.

2. Lin, Y. ; Hui, Y. N. Progress in the treatment of age-related macular degeneration: Ophthalmology, 2001, 10(6), 353.

3. Ciulla, T. A. et al. Age-related macular degeneration: a review of experimental treatments. Surv Ophthalmol. 1998, 43, 134-146.

4. O'Reilly, M. S.; Boehw, T. ; Shing, Y. et al. Endostatin: an endogenous inhabitor of angionesis and tumor growth . Cell, 1997, 88(2), 277-85.

5. Kwak, N. et al. VEGF is a major stimulator in model of choroidal neovacularization. Invest. Ophthalmol. Vis. Sci. 2000, 41, 3158-3164. 
6. Dhanabal, M.; Volk, R.; Ramchandran, R. et al. Cloning, expression, and in vitro activity of human endostatin. Biochem. Biophys. Res. Commun. 1999, 258, 345-52.

7. Mori. K.; Ando, A.; Gehlbach, P. et al. Inhibition of choroidal neovascularization by intravenous injection of adenoviral vectors expressing secretable endostatin. Am. J. Pathol. 2001, 159, 313-20.

8. Dobi, E.T.; Puliafito, C.A.; Destro, M. A new model of experimental choroidal neovascularization in the rat. Arch. Ophthalmol. 1989, 107(2), 264-9.

9. Isbida, K.; Yosbimura, N.; Mandai, M. et al. Inhibitory effect of TNP-470 on experimental choroidal neovascularization in a rat model. IOVS, 1999, 40(7),1512-9.

10. Edelman, J.; Castro, M. Quantitative image analysis of laser-induced choroidal neovascularization in rat. Exp. Eye Res. 2000, 71, 523-33.

11. Hikichi, T.; Mori, F.; Takamiya, A. et al. Inhibitory effect of bucillamine on laser-induced choroidal neovascularization in rats. Curr. Eye Res. 2002, 24(1), 1-5.

12. Kuroki, A.M.; Bhutto, I.A.; Kitaoka, T. et al. Natural course of experimental choroidal neovascularization: three-dimensional study with corrosion cast and scanning electron microscope. Ophthalmic Res. 2002, 34(4), 200-5.

13. Shang, Q.L.; Ma, J.X.; Wei, J.S. et al. Experimental study on endostatin inhibition of choroidal neovascularization. Chinese Journal of Ophthalmology, 2004, 40(4), 266-71.

14. Dhanabal, M.; Ramchdram, R.; Waterman, M.J.F. et al. Endostatin induce endothelial cell apoptosis. J. Biol. Chem. 1999, 274(17), 11721-6.

15. Yamaguchi, N.; Anand-Apte, B.; Lee, M. et al. Endostatin inhibits VEGF-induced endothelial cell migration and tumor growth independently of Zinc binding. EMBO J. 1999, 18(16), 4414-23.

16. Miosge, N.; Sasaki, T.; Timpl, R. Angiogenesis inhibitor endostatin is a distinct component of elastic fibers in vessel walls. FASEB J. 1999, 13(13), 1743-50.

17. Jia, Y.H.; Dong, X.S.; Wang, X.S. Effects of endostatin on expression of vascular endothelial growth factor and its receptors and neovascularization in colonic carcinoma implanted in nude mice. World J. Gastroenterol. 2004, 10(22), 3361-4.

18. Kim, Y.M.; Jang, J.W.; Lee, O.H. et al. Endostatin inhibits endothelial and tumor cellular invasion by blocking the activation and catalytic activity of matrix metalloproteinase. Cancer Res. 2000 , 60(19), 5410-3.

19. Yi, X.; Ogata, N.; Komada, M. et al. Vascular endothelial growth factor expression in choroidal neovascularization in rats. Graefes. Arch. Clin. Exp. Ophthalmol. 1997, 235(5), 313-9.

20. Shen, W.J.; Yu, M.J.T.; Barry, C.J. et al. Expression of cell adhesion molecules and vascular endothelial growth factor in experimental choroidal neovascularisation in the rat. $\mathrm{Br} . J$. Ophthalmol. 1998, 82, 1063-1071.

21. Wada, M.; Ogata, N.; Otsuji, T. et al. Expression of vascular endothelial growth factor and its receptor (KDR/flk-1) mRNA in experimental choroidal neovascularization. Curr. Eye Res. 1999, 18(3), 203-13.

22. Takebana, Y.; Kurokawa, T.; Kitamura, T. et al. Suppression of laser-induced choroidal neovascularization by oral tranilast in the rat. Invest. Ophthalmol. Vis. Sci. 1999, 40(2), 459-66.

(C) 2007 by MDPI (http://www.mdpi.org). Reproduction is permitted for noncommercial purposes. 